\title{
Serratus Anterior Plane blocks for pain control in anticoagulated patients with traumatic rib fractures. A case series.
}

\author{
A.J. Watts (ST7), D. Tong (Cons). King's College Hospital, Anaesthetics \\ Department, London, United Kingdom.
}

- Blanco et al. first describe Serratus Anterior plane (SAP) block. Anaesthesia (2013) 68 1107-213

- Suggested benefit for patients who are anticoagulated and epidural contraindicated Anaesthesia (2014)69,928-938

- BJA Education June 2016 described benefit for traumatic injuries and benefits in rib fractures as part of multimodal analgesic approach.

- We describe a series of cases in which a SAP block has been used in a London Major Trauma Centre in the emergency setting where patients' concurrent treatment has required anticoagulation.

- Traumatic rib fracture cases referred to emergency theatre for SAP blocks, in a major trauma centre, were screened for concurrent anti-coagulation use over a 3 month period.

- 5 cases were identified where Serratus Plane blocks were conducted with concurrent anticoagulation.

- AAGBI describes fascial blocks with a relative risk in anticoagulated patients at the lower end of the scale and addendum stating Ultrasound guidance in expert hands reduces risk of vasculature puncture, hence may be safe in altered coagulation. These cases suggest benefit that outweigh a risk. which was mitigated through confirmation of vasculature absence using colour doppler.

- We ran a quality improvement project to provide 24 hour access to SAP blocks and continuous infusions to benefit all traumatic rib fracture patients.

- The project improved appropriate and timely recognition, referral and receipt of Serratus Anterior Plane blocks with catheter infusions, through teaching, theatre availability and pharmacy protocols.

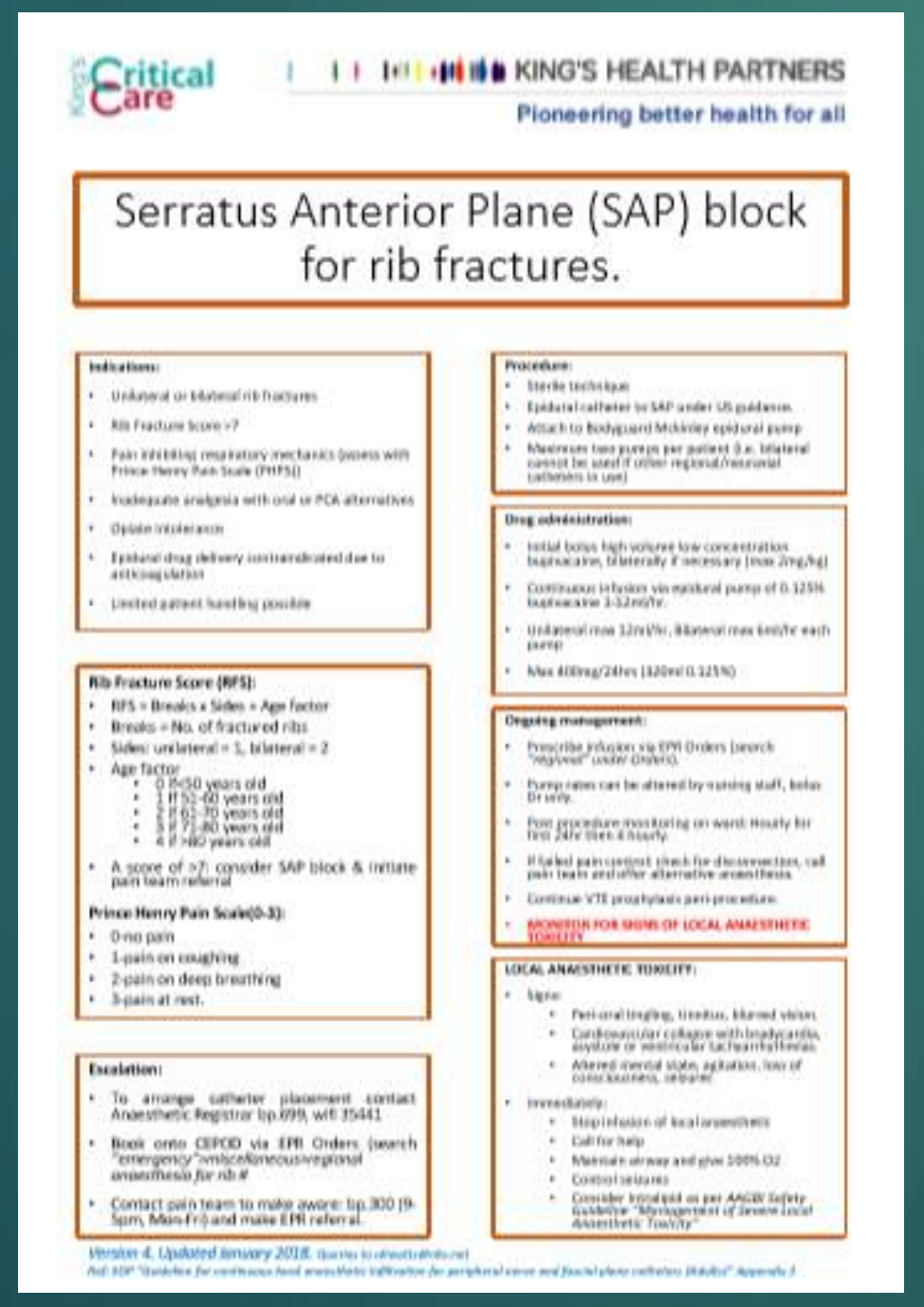

Fig.3.

King's

SAP

protocol

- W. Harrop-Griffiths et al. 2013 AAGBI Guidelines: patients with abnormalities of coagulation Anaesthesia

- May et al. (2016) "Rib fracture management" BJA education 16(1) pp26-32

- Blanco et al. (2013) "Serratus plane block: a novel ultrasoundguided thoracic wall nerve block" anaesthesia 68(11)pp1107-1113

- Letters to the Editor. Anaesthesia (2014)69,928-938
- $20 \mathrm{M} 130 \mathrm{~kg}$ RTA ejected from vehicle. Cardiac arrest at scene>ROSC.

- I\&V, Bilat thoracostomies and Code RED HEMS transfer to Kings. Further arrest in hospital $>$ ROSC

- Post extubation D2: aspiration pneumonia, painful Right sided rib \#2\&3, deformity4-6, opioid induced hypoventilation. CTPA demonstrates large R segmental PE, to start iv Heparin

- Received SAP: Needle path checked with colour flow doppler prior to placement

- $\quad 40 \mathrm{ml} 0.5 \%$ laevo-bupivacaine to plane, catheter inserted with $4 \mathrm{~cm}$ in plane. $0.25 \%$ laevo-bupivacaine at $5-10 \mathrm{ml} / \mathrm{hr}$

- Pt well orientated\& mobilising as $\mathrm{R}$ side chest pain much improved

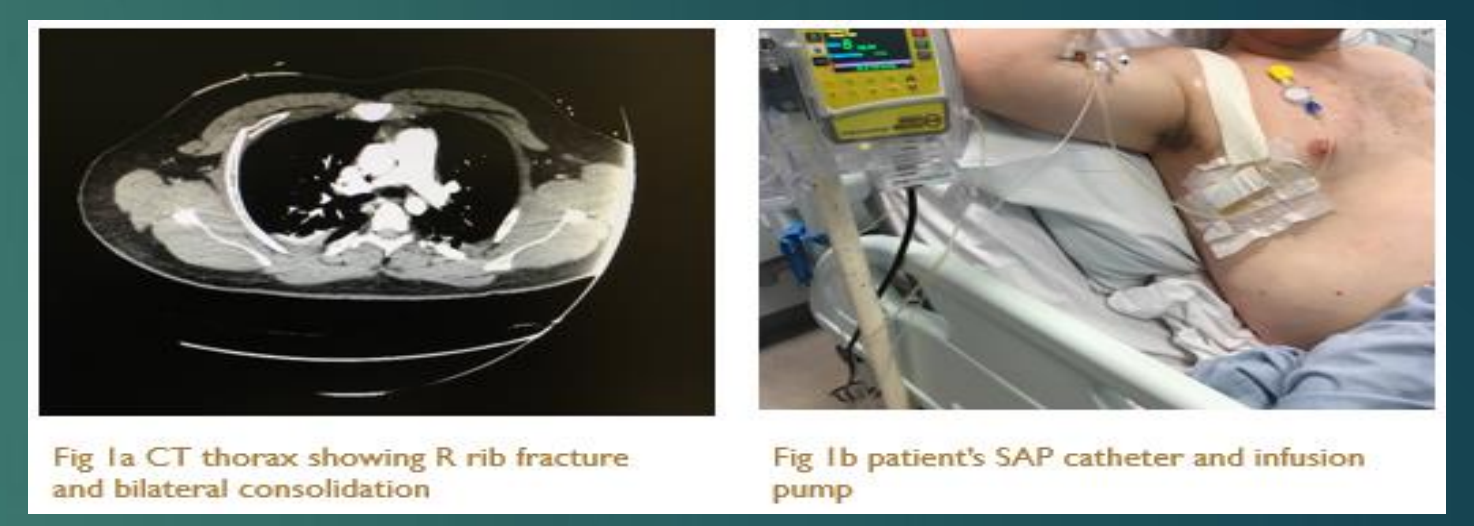

- $\quad 19 \mathrm{M}$ falls skateboarding onto R side.

- Develops large retroperitoneal haematoma displacing kidney due to ruptured incidental large vascular angiomyolipoma arising from R medial kidney.

- Initial $\mathrm{R}$ renal artery embolization, associated with severe pain. Given iv Heparin during procedure.

- Followed immediately by transfer to emergency theatre for open $\mathrm{R}$ nephrectomy. Incision umbilicus to xiphisternum and to right sub costal margin.

- Unsuitable for epidural so given SAP block 30ml $0.25 \%$ bupivacaine for initial post op-pain relief to excellent effect.

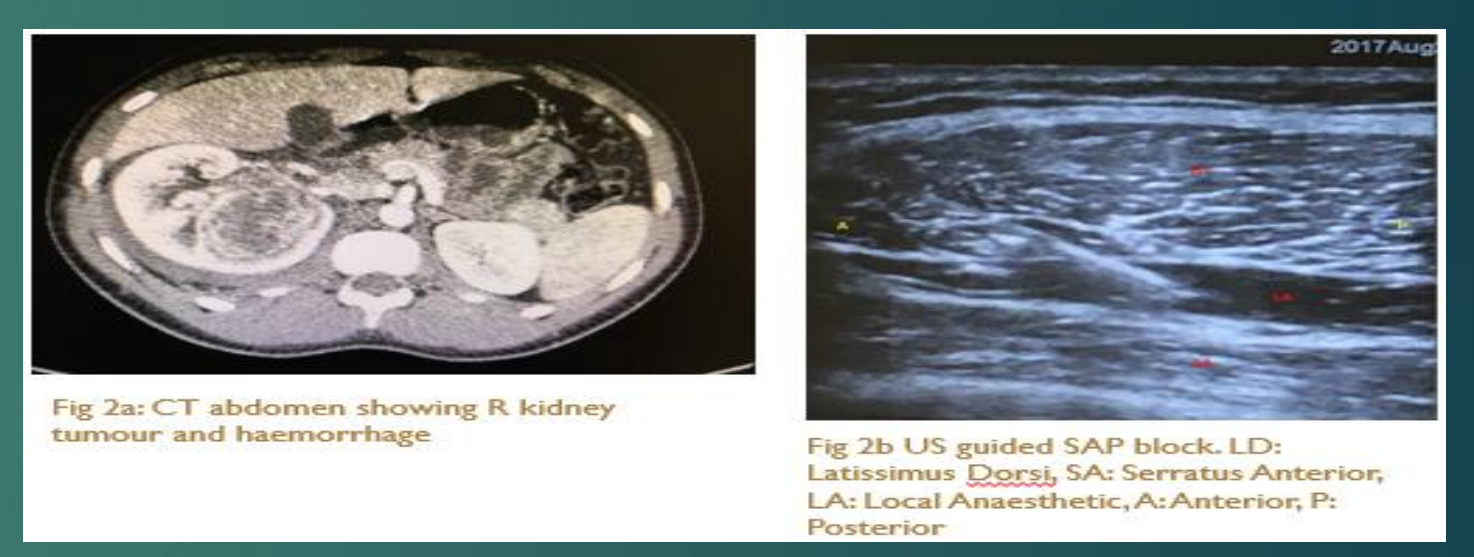

- $83 \mathrm{~F}$ with sternum\# and R7-9 rib \#, on LMWH in lieu of warfarin

- for AF.

- Poor compliance with PCA due to confusion

- SAP catheter inserted and used with regular bolusing for 3 days

- LMWH given one hour after procedure without complication.

- $\quad$ 92F Fall onto side of bath. Left rib 6-8\#, L femur\#, pubic rami\#.

- D4 post IM nailing increased work of breathing. Treated with Optiflow and listed for SAP. On warfarin for AF, INR 2.

- SAP initial bolus $30 \mathrm{ml} 0.25 \%$ and infusion commenced. Ultrasonography confirmed absence of thoracodorsal artery in needle trajectory.

- $83 \mathrm{~F}$ pedestrian v. reversing HGV.

- Bilateral rib fractures 1-10 including $\mathrm{R}$ sided 3 rib flail, pelvic fracture and ruptured bladder.

- PMH: AF on warfarin and dementia.

- Bilateral SAP blocks and catheter inserted simultaneous to resuscitation and coagulation modifiers. Doppler used to exclude arterial presence in needle trajectory for SAP.

- $\quad 30 \mathrm{ml}$ and $20 \mathrm{ml} 0.125 \%$ bupivacaine to right and left respectively and infusion via catheter continued. 